\title{
Prevalencia de anemia infantil y factores socioculturales de las usuarias del Programa Juntos, distrito de Pampas
}

Prevalence of childhood anemia and sociocultural factors of the users of the Juntos Program, Pampas

Olivia, Alarcón Sotol,

Froy Solís Luis2

Dennis Victoria Quinto3

RECIBIDO: MARZO 12017

ACEPTADO: MAYO 142017

\footnotetext{
${ }^{1}$ Enfermera, Maestra en Gestión del Desarrollo Social, Responsable de la estrategia sanitaria de crecimiento y desarrollo CRED- Hospital de Pampas - Tayacaja Huancavelica Perú, olita1506@hotmail.com

${ }^{2}$ Sociólogo, Magíster en Administración Pública y Gobierno, Docente de la Universidad Continental - Huancayo, yorf19@hotmail.com

${ }^{3}$ Sociólogo, Estudios de Maestría en Gestión del Desarrollo Social, Consultor estadístico,

dvictoriaq@hotmail.com
}

Socialium revista científica de Ciencias Sociales, Vol 1 - No. 1, julio a diciembre 2017, Pag 50-58

DOI: https://doi.org/10.31876/sl.v1i1.3 


\section{Resumen}

Esta investigación tuvo por objetivo medir el nivel de influencia de los factores socioculturales de las usuarias del Programa Juntos en la prevalencia de anemia infantil en el Hospital de Pampas (Tayacaja, Huancavelica). Se realizó una investigación de tipo básico, nivel descriptivo, diseño no experimental transversal y con un enfoque metodológico cuantitativo. Se aplicó un cuestionario a 36 madres usuarias del Programa Juntos; los hijos de 16 de estas presentan prevalencia de anemia, y los de los 16 restantes, no. Al $95 \%$ de confianza estadística, los resultados obtenidos fueron: el $82 \%$ de las madres cuyos hijos tienen anemia son menores de 30 años; los hijos del $71 \%$ de madres, que en su mayoría proceden de la zona rural, presentan prevalencia de anemia infantil; el $68 \%$ de las madres tienen conflictos con los padres de sus hijos; solo el $23 \%$ de las madres que tienen acceso a servicios públicos con agua potable son menos recurrentes a la prevalencia de anemia; el $76 \%$ de ellas participan en otros programas sociales, y $77 \%$ de las mimas confían en el sistema de salud. Se concluye que los factores sociales en las usuarias del programa juntos del hospital de Pampas, Tayacaja influyen directa y significativamente en la prevalencia de la anemia infantil, el comportamiento de la madre, sus condiciones de vida, la iteración con su menor hijo (a) y con el grupo de personal que socializa cotidianamente, genera un estilo de vida que no contribuyen en la superación del diagnóstico de anemia y pasan hacer prevalentes, afectando directamente al desarrollo físico y emocional del infante, esta situación compromete el futuro del mismo, propiciando un escenario que se torna negativo para el desarrollo humano, la generación del capital social, y en consecuencia para el desarrollo social. Los factores culturales en las usuarias del programa juntos del hospital de Pampas, Tayacaja influyen directa y significativamente en la prevalencia de la anemia infantil, los hábitos y las costumbres cotidianas de comportamiento de la madre en sus entorno, los elementos que pautan la convivencia con su menor hijo (a), a genera una cultura de convivencia que no contribuye a la superación del diagnóstico de anemia más por el contrario se presentan como prevalentes, esta forma de ver la vida la valoración de la situación de la salud están influenciadas por la forma de concebir el mundo, su idiosincrasia no permite con facilidad asumir la situación de importancia y prioridad de la 
atención al menor con hábitos alimenticos y de higiene más adecuado al tratamiento de la anemia infantil.

Palabras clave: prevalencia de la anemia, factor social, factor cultural.

\begin{abstract}
This research aimed to measure the level of influence of sociocultural factors of the users of the Juntos Program on the prevalence of childhood anemia at the Pampas Hospital (Tayacaja, Huancavelica). An investigation of basic type, descriptive level, transversal non-experimental design and with a quantitative methodological approach was carried out. A questionnaire was applied to 36 mothers using the Together Program; the children of 16 of these have a prevalence of anemia, and those of the remaining 16, no. At $95 \%$ statistical confidence, the results obtained were: $82 \%$ of mothers whose children have anemia are under 30 years of age; the children of $71 \%$ of mothers, who mostly come from the rural area, have a prevalence of childhood anemia; $68 \%$ of mothers have conflicts with their children's parents; Only $23 \%$ of mothers who have access to public services with drinking water are less recurrent to the prevalence of anemia; $76 \%$ of them participate in other social programs, and $77 \%$ of the same trust in the health system. It is concluded that the social factors in the users of the program together of the Pampas hospital, Tayacaja directly and significantly influence the prevalence of childhood anemia, the mother's behavior, her living conditions, the iteration with her youngest child. and with the group of personnel that socialize every day, it generates a lifestyle that does not contribute to overcoming the diagnosis of anemia and they become prevalent, directly affecting the physical and emotional development of the infant, this situation compromises its future, promoting scenario that becomes negative for human development, the generation of social capital, and consequently for social development. Cultural factors in the users of the program together at the Pampas, Tayacaja hospital directly and significantly influence the prevalence of childhood anemia, the habits and daily habits of the mother's behavior in her surroundings, the elements that guide the coexistence with her Younger child, a generates a culture of coexistence that does not contribute to the overcoming of the diagnosis of anemia but on the contrary they are presented as prevalent, this way of seeing life, the assessment of the health situation are influenced by the way If you conceive the world, your idiosyncrasy does not allow you to easily assume the situation of importance and priority of child care with eating habits and hygiene more appropriate to the treatment of childhood anemia.
\end{abstract}

Keywords: prevalence of anemia, social factor, cultural factor. 


\section{Introducción}

Organización Mundial de la Salud (OMS), califica a la anemia infantil como el principal problema de salud que afronta el mundo de hoy; El $43.6 \%$ de niños de entre 6 y 35 meses padecen esta afección en todo el país de acuerdo con la Encuesta Demográfica y de Salud Familiar (ENDES 2017); en el 2016, la cifra era también de $43.6 \%$, y en el 2015 , de $43.5 \%$. Las estrategias implementadas a nivel nacional como políticas públicas no han podido reducir estos niveles de anemia infantil. Según el Instituto Nacional de Estadística e Informática (INEI), las regiones que reportan mayor presencia de esta enfermedad son Puno (75.9\%), Loreto (65.5\%), Ucayali (59.1 \%), Pasco (58 \%) y Huancavelica (42.5\%).

Estas cifras son propias de poblaciones vulnerables, donde la anemia afecta directamente las etapas de desarrollo físico y mental de los niños. Esta afección se vuelve prevalente cuando luego del tratamiento inicial, el menor no supera el nivel mínimo requerido de hemoglobina y se mantiene con un déficit de hierro en el organismo, comprometiendo a largo plazo el desarrollo de sus habilidades para el aprendizaje. En consecuencia, el futuro de un ciudadano libre, que disfruta de una vida plena y contribuye al desarrollo de su comunidad, corre riesgo.

La presente investigación es de importancia para las ciencias sociales, debido a que, en la provincia de Pampas, así como en todo el Perú, los efectos socioambientales influyen la prevalencia de la anemia en los niños. En tal sentido, este estudio aborda la problemática en mención desde un enfoque de la sociología de la salud.

Sammartino (2010), a través de entrevistas semiestructuradas y abiertas aplicadas a profesionales de la salud y no profesionales que laboran en centros de salud, así como a gestantes e infantes, concluyó que los equipos de salud deben ser capacitados para poder transmitir a las madres la informacion y los saberes que podrían incrementar y mejorar sus conocimientos sobre la enfermedad y su tratamiento.

Oyola y Valdez (2013) señalan que los determinantes sociales de la salud han mejorado en los ultimos años, pero aún hay indicadores en los que se debe seguir trabajando, como en la alfabetización, el acceso a saneamiento básico (agua), el parto institucional, la cobertura de inmunización y el aseguramiento de los infantes menores de cinco años. Además, son necesarios el fortalecimiento de competencias del talento humano en salud así como la invesrsion del Estado peruano en servicios que mejoren la calidad de vida. 
Junco (2015) infiere en su investigación, que los factores determinanates que limitan la efectividad del plan de implementación de micronutrientes están reforzados por otros factores como la intervención educativa, las barreras culturales y lingüísticas de las madres y la débil participación de los promotores de la salud.

Casavilca y Egoávil (2017) refieren que la anemia es un problema de salud pública, cuyos factores principales son la falta de una buena dieta y de un adecuado control prenatal en madres gestantes. Asimismo, sostienen que la anemia tiene relación significativa con el parto por cesárea.

Los resultados reportaron la prevalencia de anemia por déficit de hierro en niños de entre 6 y 23 meses de edad, anteriormente sanos, pertenecientes a una zona urbana y de clase socioeconómica media-baja.

La investigación, buscó responder a la pregunta: ¿̇cuál es la influencia de los factores socioculturales de las usuarias del Programa Juntos en la prevalencia de anemia infantil en el Hospital de Pampas (Tayacaja)? Por ende, el objetivo de la investigación fue medir el nivel de influencia de los factores socioculturales de las usuarias del Programa Juntos en la prevalencia de anemia infantil en el Hospital de Pampas. La hipótesis planteada fue que los factores socioculturales de las usuarias del Programa Juntos influyen directa y significativamente en la prevalencia de anemia infantil en el Hospital de Pampas.

Con este estudio, se contribuye al enriquecimiento de conocimientos de la sociología de la salud.

\section{Materiales y métodos}

El tipo de investigación fue básica, ya que, con los resultados hallados, se contribuye al enriquecimiento del conocimiento respecto a la prevalencia de anemia infantil y los factores socioculturales en nuestro país. El nivel de investigación fue descriptivo, pues pretende medir el nivel de influencia de dichos factores en la prevalencia de anemia infantil en el Hospital de Pampas. El diseño de investigación fue no experimental transversal descriptivo, con enfoque metodológico cuantitativo.

El recojo de información se realizó a través de un cuestionario aplicado a 32 madres usuarias del Programa Juntos del Hospital de Pampas. 


\section{Resultados}

Primero, es necesario mencionar que las condiciones sociales son, según Ramírez (2012), las circunstancias, situaciones o estados que afectan la vida, el bienestar y las relaciones de los seres humanos en la comunidad. Así también, "seguridad social" es un término relacionado a la prestación médica como a los actos de las personas que buscan el bienestar social.

\section{Factores sociales influentes}

Así, la hipótesis de investigación indica que la prevalencia de anemia infantil y los factores sociales generados son: madre soltera, grado de instrucción, lugar de procedencia, ingreso familiar de la madre y la participación en otros programas sociales.

A continuación, se presentan los principales resultados hallados en el proceso de investigación.

Tabla 1: Factores sociales y la prevalencia de anemia infantil

\begin{tabular}{lll}
\hline Factor social & $\gamma$ & Valor P \\
\hline Madre soltera & 0,750 & 0,003 \\
Grado de instrucción de la madre & $-0,802$ & 0,001 \\
Lugar de procedencia rural de la madre & 0,628 & 0,029 \\
Ingreso familiar de la madre & $-0,724$ & 0,008 \\
Conflictos de la madre con el padre de sus hijos & 0,774 & 0,002 \\
Tiempo como beneficiaria del Programa Juntos de la madre & 0,677 & 0,011 \\
Participación en otros programas sociales de la madre & 0,802 & 0,001 \\
\hline
\end{tabular}

En la tabla 1 se muestra que los valores $P$ de los factores sociales son menores que el nivel de significación habitual de 0.05, por ello, al $95 \%$ de confianza estadística, se acepta que existe relación significativa entre los factores sociales de las madres y la prevalencia de anemia infantil en el Hospital de Pampas.

\section{Factores culturales influentes}

Asimismo, la hipótesis indica que la prevalencia de anemia infantil y sus factores culturales influentes son: tiempo de conservación del agua de consumo almacenada; edad de consumo de comidas sólidas del niño; consumo de sangre y vísceras; administración del medicamento según la prescripción médica; creencia de que la anemia es una enfermedad grave que afectará el futuro del niño; creencia de que el consumo de medicamentos impedirá que el niño sea inteligente; profesión de la religión católica por la madre; creencia de que la anemia es un castigo divino; 
comunicación en quechua; existencia de violencia familiar, y frecuencia de consumo mensual de bebidas alcohólicas.

Se observa que los valores $\mathrm{P}$ de los factores culturales, salvo la creencia de que la anemia es un castigo divino, son menores que el nivel de significación habitual de 0.05; por ello, al $95 \%$ de confianza estadística, se acepta que existe relación significativa entre los factores culturales de las madres y la prevalencia de anemia infantil en el Hospital de Pampas. Por lo tanto, se concluye que existen factores culturales de las madres beneficiarias del Programa Juntos que influyen en la prevalencia de anemia infantil en el Hospital de Pampas.

\section{Discusión}

Los resultados de esta investigación comprueban las hipótesis propuestas. Se afirma que los factores socioculturales de las madres beneficiarias del Programa Juntos influyen directa y significativamente en la prevalencia de anemia infantil.

La anemia, según el Minsa (2017), es un trastorno en el que el número de glóbulos rojos circulares en la sangre se ha reducido y es insuficiente para satisfacer las necesidades del organismo. UNICEF (2018) refiere que la anemia infantil es un problema nutricional que afecta a niños menores de tres años, limitando su capacidad física e intelectual.

En el Perú, la anemia está considerada como una enfermedad que se presenta debido al poco consumo de alimentos ricos en hierro, además la reducción de la lactancia materna exclusiva, las mujeres embarazadas quienes no tienen una alimentación saludable. Asimismo, Casavilca y Egoávil (2017) indican que se trata de un problema de salud pública, teniendo en cuenta que "salud" está definida, según la OMS, como la disponibilidad de acceder a los servicios de salud, buenas condiciones de trabajo, una adecuada vivienda y a una buena alimentación. Si bien el concepto de salud abarca libertades y derechos de cada persona, se ha observado que, en el Hospital de Pampas, existen factores socioculturales de las madres usuarias del Programa Juntos que condicionan la prevalencia de anemia infantil.

La prevalencia de anemia infantil en los hijos de las usuarias del Programa Juntos obedece también a un conjunto de factores sociales que influyen en ella significativamente; tal es el caso de la edad de la madre, su estado civil, grado de instrucción, número de hijos a cargo, demografía, trabajo, ingreso económico, régimen de tenencia de tierra, beneficio de otros programas del Estado, conflictos con la pareja, gasto diario en la alimentación, entre otros. 
De otro lado, los factores culturales que influyen significativamente en la prevalencia de anemia infantil son: la falta de servicios básicos; inadecuadas prácticas de higiene; la poca presencia y capacitación de personal de salud; la falta de información sobre lactancia materna; la falta de un buen hábito de consumo de alimentos nutritivos; ineficientes modelos de monitoreo en el consumo de medicamentos para contrarrestar la anemia; creencias; la religión; el idioma, y la violencia familiar. Al respecto, Casavilca y Egoávil (2017) refieren que los principales factores para la prevalencia de anemia son la falta de una buena dieta alimenticia y un adecuado control prenatal en madres gestantes.

En ese sentido, Sammartino (2010) resalta que las madres deben recibir información precisa acerca de la anemia y su tratamiento.

Oyola, Vilchez, Napanga y Valdez (2013), en su artículo Análisis de los determinantes sociales de la salud en el Vraem, indican que, para mitigar los problemas de salud pública el Estado debe invertir más con políticas estatales, como saneamiento básico, alfabetización, parto institucional, la cobertura de inmunizacion y el aseguramiento de los infantes menores de cinco años, asi como el fortalecimiento de competencias del talento humano en salud entre otros. Weber (1944) sostiene que tales políticas deben emanar de un Estado moderno.

Fernández (2017) señala que es derecho fundamental de los niños la lucha contra la mortalidad, independientemente donde estén situados o en qué condiciones se encuentren; este debe ser vinculado con el derecho a la vida, la alimentación, el trabajo, la educación y a la igualdad. En esa línea, el Ministerio de Inclusión y Desarrollo Social (Midis) pone en funcionamiento el Programa Juntos, a través del cual se entregan subsidios a familias consideradas pobres y pobres extremas, para promover la inserción escolar y una buena salud. Con base en la teoría del desarrollo social, este programa actúa como un garante que busca equilibrar los derechos de los grupos más vulnerables del país.

Por otra parte, Sammartino (2010) concluyó que los equipos de salud deben capacitarse para poder transmitir a las madres la información y los saberes sobre la prevención y el tratamiento de la anemia infantil.

El Programa Juntos se focaliza en la población de entre 0 y 5 años y en las madres y gestantes. Debemos destacar que en el Hospital de Pampas, las madres no llevan a sus hijos puntualmente a sus controles de crecimiento y desarrollo (CRED) ni a sUs controles preventivos de salud; además, estos no consumen sus complementos nutricionales, como la papilla, ni los suplementos vitamínicos recetados. Las conductas inadecuadas de las madres dependen también de los factores sociales y culturales para la prevalencia de anemia infantil. Debería haber un compromiso 
voluntario de las madres usuarias del Programa Juntos para llevar a sus hijos a sus controles y cumplir las instrucciones del personal de salud del Hospital de Pampas.

\section{Referencias bibliográficas}

Casavilca, K., y Egoávil, J. (2017). Factores en riesgo y anemia en el post parto en el Hospital Nacional Edgardo Rebagliati Martins durante el año 2016 (Tesis de pregrado). Universidad Nacional del Centro del Perú, Huancayo.

Fernandez, D. (20 de Octubre de 2017). El derecho delos niños a la salud. Guia infantil, 2. Obtenido de Guia infantil: https://www.guiainfantil.com/articulos/educacion/derechos-del-nino/el-derechode-los-ninos-a-la-salud/

Junco, J. (2015). Identificación de los factores que contribuyen y limitan la efectividad del programa de suplementación con micronutrientes en la reducción de la anemia de niños menores de tres años del ámbito rural de Vinchos, Ayacucho (Tesis de maestría). Pontificia Universidad Católica del Perú, Lima. Recuperada de http://tesis.pucp.edu.pe/repositorio/bitstream/handle/123456789/6650/JUNCO_GUIL LERMO_JORGE_IDENTIFICACION_FACTORES.pdf? sequence=1 \&isAllowed=y

Juntos. (s. f.). Programa Nacional de Apoyo Directo a los más Pobres. Recuperado de https://plataformacelac.org/programa/186

Galindo, M.L. (2012). Condiciones sociales, culturales y económicas de las maras en Centroamérica. Un estudio comparativo: jóvenes y violencias en Bogotá. Revista Logos, 3 (2), 21. Recuperado el 14 de octubre de 2018, de http://www.redalyc.org/html/5177/517751762002/

Minsa. (2017). Norma técnica. Manejo terapéutico y preventivo de la anemia en niños, adolecentes, mujeres gestantes y puérperas. Lima: Minsa. Recuperado de http://www.ins.gob.pe

Oyola, A., Vilchez, A., Napanga, E., y Valdez, W. (2013). Análisis de los determinantes sociales de la salud en el Vraem. Revista Peruana de Epidemiología, 2, 02-05. Recuperado de http://www.redalyc.org/html/2031/20312945901 1/

Sammartino, G. (2010). Representaciones culturales acerca de la anemia y la suplementación con hierro. Arch Argent Peditr, 108, 401-408. Recuperado de https://www.sap.org.ar/docs/publicaciones/archivosarg/2010/v108n5a05.pdf 
